\title{
Determinants of Tuberculosis among HIV infected adults in Horro Guduru Wollega Zone, West Ethiopia: A facility-based case-control study
}

Zelalem Belay ${ }^{1}$, Belachew Etana², Mekdes Tigistu Yilma ${ }^{2}$

\section{Address}

${ }^{1}$ Nekemte Health Sciences College, Nekemte, Ethiopia

${ }^{2}$ Department of Public Health, Institution of Health Science, Wollega University, Nekemte, Ethiopia

Email addresses

ZB: Zalalambelay@gmail.com

BE: ba.etana@gmail.com

MT: mekdestigistu10@gmail.com

\section{Corresponding Author:}

Zelalem Belay 


\begin{abstract}
Background: Tuberculosis is a contagious air born disease caused by Mycobacterium tuberculosis species and the leading causes of morbidity and mortality among people living with HIV/AIDS worldwide. Globally, it causes ill-health among millions of people living with HIV/AIDS each year.
\end{abstract}

Objective: To identify determinants of Tuberculosis among HIV infected adults in public health facilities, in Horro Guduru Wollega Zone, 2019.

Methods: Unmatched case-control study was conducted among 127 cases and 255 controls which were selected using systematic random sampling technique. Data were collected by record review and through face to face interview. Then, analysis was done using SPSS version 25.To identify determinants of tuberculosis multivariable logistic regression was employed. Adjusted odds ratio was calculated with $95 \%$ CI to show strength of association and P-value $<0.05$ was used to declare statistical significance.

Results: - A total of 127 cases and 255 controls were participated into the study with $97.2 \%$ response rate. Aged $\geq 35$ years $[\mathrm{AOR}=2.63,95 \% \mathrm{CI}(1.29,5.36)]$, alcohol consumption $[\mathrm{AOR}=3.34,95 \% \mathrm{CI}(1.68,6.99)]$, Having CD4 cells <200 [AOR=3.67, 95\% CI $(1.5,9.1)]$, Having Haemoglobin $<11 \mathrm{~g} / \mathrm{dl} \quad[\mathrm{AOR}=3.77,95 \% \mathrm{CI}(1.84,7.72)]$, Imprisoned in the past 2 years $[\mathrm{AOR}=4.22,95 \% \mathrm{CI}(1.56,11.37)]$, Living with TB patients in the same house $[\mathrm{AOR}=14.97,95 \%$ CI(1.99, 21.63)] were factors associated with TB among HIV infected adults. Importantly, using Cotrimoxazole prophylaxis $[\mathrm{AOR}=0.193,95 \% \mathrm{CI}(0.81,0.46)]$ had a protective effect from acquiring TB.

Conclusion and Recommendation: Being in old age, alcohol use, Chewing khat, Having CD4cells <200, Advanced WHO clinical stage, Hgb <11g/dl, Imprisoned in the past 2 years, History of previous TB and living with TB patients in the same house were factors associated with TB/HIV co-infection. Using Cotrimoxazole had a protective effect from acquiring TB/HIV co-infection. For most of these determinants interventions can be made at individual and institutional levels, whereas, some factors need societal level integrations.

Key words: TB /HIV co- Infection, Tuberculosis, determinants, West Ethiopia. 


\section{Background}

Tuberculosis is one of the most sever and extremely contagious air born disease caused by Mycobacterium Tuberculosis including Mycobacterium bovis and Mycobacterium Africanum and affect every organ of human body. The commonest TB infection, causes of morbidity and mortality in people infected with HIV/AIDS is pulmonary tuberculosis. About 1.7 billion people (23\% of world population) have latent TB and 5\%-10\% of people with latent TB will develop active TB at some point in their lives $(1,2,3)$.

$\mathrm{HIV}$ is one of the risk factors for the development of TB and the double TB/HIV co-infection is a major challenge to control the spread of TB which continues to sustain the TB epidemic. Risk of developing TB is high among HIV infected adults; It is estimated to be 26- 31 times greater in people living with HIV than among those without HIV infection(4).

Tuberculosis is one of the world's most common causes of death in era of Human Immunodeficiency Virus(5). According to 2018 WHO tuberculosis report, 464633 cases of TB among people living with HIV were notified and majority of the people live in developing countries where resources are limited (6).

In Sub-Saharan Africa including Ethiopia, the incidence of TB is high in adults receiving HAART than in HIV negative adults due to their low level of immunity $(2,4)$. Factors like sociodemographic, clinical, behavioral and health facility related factors were indicated as a risk of TB/HIV co-infection, but this factors varies contextually and from place to place indicating that some people with HIV/AIDS are infected with TB while other don't (7-9). This shows that HIV infection is not the only factor for being infected with TB and there are other determinant factors which varies contextually and from one setting to other settings $(5,9)$. 


\section{Methods}

\section{Study setting and population}

The study was conducted in Horro Guduru Wollega Zone of Oromia Regional state. The zone has twelve Woredas and 37 health facilities of which twelve health facilities providing ART services. There are twelve health care institutions (one hospital and eleven health centers) providing ART services in the zone during the study period. Among these, one hospitals and five health centers were selected by simple random sampling technique. There are 3,022 people following ART care in the Zone, among this 136 of them were children under 15 years and 2886 were above 15 years, 936 were TB/HIV co-infected before data collection. The study was conducted from August 15 to October 15/2019.

In this study, a case was defined as those adults having confirmed TB/HIV co-infection by health professional and eligible for the study during data collection period control was defined as Adult HIV patients free of TB decided by health professional and eligible for study during data collection time.

The source population of the cases was all patients with TB/HIV co-infected among public health facilities in Horro Guduru Wollega zone while the source population for controls was all patients with HIV infected free of TB (Non TB infected patients) among public health facilities in Horro Guduru Wollega zone. All adults ( $\geq 18$ years) TB patients who had no any severe mental problems participated in the study while TB patients who were not volunteer to participate and transferred to other facilities outside of the study area, Patients whose information is incomplete on their medical record were excluded from the study.

\section{Sample size and Sampling technique}

Sample size was determined by using Epi Info 7 statistical software to determine two population proportion from the factors reviewed which gives maximum value by using $95 \% \mathrm{CI}$, power $80 \%$, control to case ratio $2: 1, \mathrm{AOR}=3.5$ which was the ratio of odds of having DM among HIV patients with TB to odds of having DM among HIV patients free of TB, prevalence of DM 
among HIV patients free of $\mathrm{TB}=4.2 \%$ and probability of having DM among HIV patients with TB was $13.4 \%(5)$.

The calculated sample was 357 (119 cases and 238controls). By adding 10\% non-response rate, the total sample size was 393 (131 cases and 262 controls). Before main study, cases ( $n=936$ ) and controls $(n=1005)$ were identified using the aforementioned case and control definitions. Sampling frame of cases and controls was prepared by using register of the patients and systematic random sampling technique was used to select cases and controls for final data collection.

\section{Data collection procedures}

Data was collected by both record review and interviewer administered questionnaire. The questionnaire was prepared in English, translated to Afan Oromo language then back translated to English to check for consistency. The questionnaire was developed by the principal investigator after reviewing different literatures.

To ensure the quality of data, a range of mechanisms was employed to address major areas of bias introduction during the data collection process. First, data collectors were trained on how to gather the appropriate information, procedures of data collection techniques and the whole contents and subject matter of the questionnaire.

A week prior to the actual data collection, the questionnaire was pre-tested on $5 \%$ of sample on 19 (6 cases and 13 controls) in Nekemte specialized Hospital among HIV infected patients. Amendments like wording, logic and skip order of the questions was made accordingly after the pre-test. The reliability test of the questionnaire was done by Cronbach's alpha and it was 0.71 .

Trained four BSc Nurses and one Health officer collected data. Additionally, five Health officers who were fluent in Afan Oromo supervised the data collection process. Daily, on site supervision by the supervisors were carried out during the whole period of data collection. At the end of each data collection day, the questionnaire was reviewed and cross checked for completeness, accuracy and consistency by the supervisors. Data was cleaned after it was entered in to the software. 


\section{Measurement procedures}

Weight was measured in light clothing and without shoes in Kilograms ( $\mathrm{Kg}$ ) using calibrated digital weighing scale at a precision of $0.1 \mathrm{~kg}$ as per recommended but not repeated measurements. Height was measured using Stadiometer in Centimeter $(\mathrm{cm})$ in erect position that the back of the head, shoulder blades, buttocks and heels make contact with the backboard at a precision of $0.1 \mathrm{~cm}$ with shoes removed as per recommended but not repeated measurements. Adherence was taken from their medical card whether they were optimally adhered to ART medication.

\section{Data analysis}

Collected data was coded and entered in to Epi-Data version 4.6 and then exported to SPSS 25 for analysis. A descriptive analysis was conducted to check for outliers and consistencies and to identify missed values for independent variables. Frequencies and cross tabulations was done to organize the data and presented using table and graph.

The association between TB/HIV co-infection and each covariate was assessed by Binary logistic regression. Variables with $\mathrm{P}$-value $<0.25$ in bivariable analysis was taken to multiple Logistic regression. Logistic regression with backward elimination method was used to control possible confounders and to identify the determinant factors associated with TB/HIV coinfection. Wealth index was analysed by Principal Component analysis (PCA) after checking all its assumptions.

Crude OR was estimated with $95 \%$ CI to show strength of association and P-value $<0.05$ was used to declare statistical significance. Goodness of fit of the model was checked using Hosmer Lemeshow test of goodness of fit considering good fit at large P-value $>0.05$. Multicollinearity was checked by using VIF and maximum value was 2.18 . 


\section{Results}

\section{Socio demographic characteristics}

A total of 382(127 cases and 255 controls) of HIV positive patients in selected Hospital and five Health centres were interviewed with response rate of $97.2 \%$. The mean age ( \pm Standard deviation) for the cases and the controls were 39.87 ( $\mathrm{SD} \pm 9.51$ ) and 36.57( $\mathrm{SD} \pm 10.44$ ) respectively. Seventy five (59.1\%) of cases and 171(67.1\%) of controls were female participants. From all of the participants, $82(64.6 \%)$ of cases and 175(68.6\%) of controls were from urban residence. Concerning the marital status of the respondents, 63(49.6\%) of cases and 152(59.6\%) of controls were married. Concerning Educational status, 49(38.6\%) of cases and 85(33.3\%) of controls were lacks formal education (Illiterate), 33(26\%) of cases and 102(40\%) of controls were attended primary education, $23(18.1 \%)$ of cases and 36(14.1\%) of controls were secondary school. 
Table 1.Socio demographic characteristics of HIV infected adults in Public Health Facilities of Horro Guduru Wollega Zone, Ethiopia, 2019.

\begin{tabular}{|c|c|c|c|c|}
\hline Variables & $\begin{array}{l}\text { Cases } \\
\mathbf{N}=127\end{array}$ & $\begin{array}{l}\text { Controls } \\
\mathbf{N}=255\end{array}$ & $\operatorname{COR}(95 \% \mathrm{CI})$ & P-Value \\
\hline \multicolumn{5}{|l|}{ Age } \\
\hline $18-34$ & $46(36.2 \%)$ & $134(52.5 \%)$ & 1 & \\
\hline$\geq 35$ & $81(63.8 \%)$ & $121(47.5 \%)$ & $1.95(1.259,3.020)$ & 0.003 \\
\hline \multicolumn{5}{|c|}{ 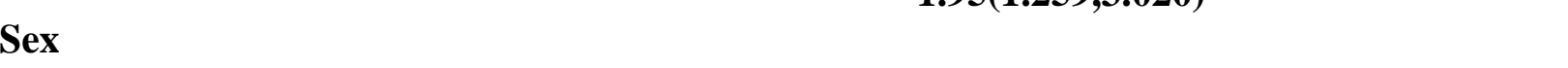 } \\
\hline Male & $52(40.9 \%)$ & $84(32.9 \%)$ & $1.41(0.909,2.191)$ & 0.124 \\
\hline Female & $75(59.1 \%)$ & $171(67.1 \%)$ & 1 & \\
\hline \multicolumn{5}{|l|}{ Residence } \\
\hline Urban & $82(64.6 \%)$ & $175(68.6 \%)$ & $0.83(0.531,1.306)$ & 0.426 \\
\hline Rural & $45(35.4 \%)$ & $80(31.4 \%)$ & 1 & \\
\hline \multicolumn{5}{|l|}{ Marital status } \\
\hline Married & $63(49.6 \%)$ & $152(59.6 \%)$ & 1 & \\
\hline Single & $9(7.1 \%)$ & $31(12.2 \%)$ & $0.7(0.315,1.556)$ & 0.382 \\
\hline Widowed & $35(27.6 \%)$ & $34(13.3 \%)$ & $2.48(1.425,4.330)$ & 0.001 \\
\hline Divorced & $20(15.7 \%)$ & $38(14.9 \%)$ & $1.27(0.686,2.35)$ & 0.447 \\
\hline \multicolumn{5}{|l|}{ Educational status } \\
\hline No formal Education & $49(38.6 \%)$ & $85(33.4 \%)$ & $0.839(0.439,1.601)$ & 0.539 \\
\hline Primary & $33(26 \%)$ & $102(40 \%)$ & $0.471(0.241,0.920)$ & 0.27 \\
\hline Secondary & $23(18.1 \%)$ & $36(14.1 \%)$ & $0.929(0.437,1.975)$ & 0.849 \\
\hline $\begin{array}{l}\text { Tertiary } \\
\text { Occupation }\end{array}$ & $22(17.3 \%)$ & $32(12.5 \%)$ & 1 & \\
\hline Employed & $13(10.2 \%)$ & $23(9 \%)$ & 1 & \\
\hline $\begin{array}{l}\text { Unemployed } \\
\text { Religion }\end{array}$ & $114(89.8 \%)$ & $232(91 \%)$ & $0.869(0.425,1.779)$ & 0.702 \\
\hline Protestant & $66(52 \%)$ & $121(47.5 \%)$ & 1 & \\
\hline Orthodox & $44(34.6 \%)$ & $121(47.5 \%)$ & $0.67(0.422,1.053)$ & 0.082 \\
\hline Catholic & $5(4 \%)$ & 0 & $2.17(0.78,4.309)$ & 0.53 \\
\hline Muslim & $12(9.4 \%)$ & $12(5 \%)$ & $1.64(0.78,4.309)$ & 0.164 \\
\hline
\end{tabular}


Table 1. Socio demographic characteristics of HIV infected adults in Public Health Facilities of Horro Guduru Wollega Zone, Ethiopia, 2019 (continued).

\begin{tabular}{|c|c|c|c|c|}
\hline \multicolumn{5}{|c|}{ Ethnicity } \\
\hline Oromo & $96(75.6 \%)$ & $204(80 \%)$ & $0.34(0.052,1.909)$ & 0.28 \\
\hline Amhara & $28(22 \%)$ & $46(18 \%)$ & $0.41(0.64,2.58)$ & 0.339 \\
\hline Tigre & 0 & $3(1.2 \%)$ & $0.43(0.78,4.309)$ & 0.28 \\
\hline Gurage & $3(2.4 \%)$ & $2(0.8 \%)$ & 1 & \\
\hline \multicolumn{5}{|l|}{ Income } \\
\hline Poorest & $27(21.4 \%)$ & $43(17.2 \%)$ & $1.16(0.589,2.28)$ & 0.669 \\
\hline Poor & $21(16.7 \%)$ & $60(23.8 \%)$ & $1.65(0.324,1.29)$ & 0.214 \\
\hline Middle & $20(15.9 \%)$ & $53(21 \%)$ & $1.69(0.345,1.405)$ & 0.313 \\
\hline Rich & $33(25.4 \%)$ & $48(19 \%)$ & $1.23(0.64,2.37)$ & 0.534 \\
\hline Richest & $26(20.6 \%)$ & $48(19 \%)$ & 1 & \\
\hline \multicolumn{5}{|c|}{$\begin{array}{l}\text { No. of family living } \\
\text { in the house }\end{array}$} \\
\hline $1-5$ & $59(46.5 \%)$ & $176(69 \%)$ & 1 & \\
\hline $6-10$ & $54(42.5 \%)$ & $70(27.5 \%)$ & $2.301(1.451,3.651)$ & $<0.001$ \\
\hline$>10$ & $14(11 \%)$ & $9(3.5 \%)$ & 4.64(1.91,11.275) & 0.001 \\
\hline
\end{tabular}




\section{Behavioral determinants of TB among HIV infected adults}

From study participants, Eighty (63\%) of cases and 76(29.8\%) of controls has history of ever consumption of alcohol. Forty one (32.3\%) of participants from cases and ten (3.9\%) from controls had smoking history. Among those who had ever smoked, 21(16.5\%) of cases and four controls smoke now. Regarding khat chewing, 45(35.4\%) of cases and 19(7.5\%) controls had ever chewed khat but, $82(64.6 \%)$ of cases and 236(92.5\%) of controls never chewed khat. Among all participants, 43(33.9\%) of cases and 20(7.8\%) of controls had Prisoned in the past 2 years but $84(66.1 \%)$ of cases and 235(92.2\%) controls hadn't Prisoned. 
Table 2.Behavioral determinants of TB among HIV infected adults in public health facilities of Horro Guduru Wollega Zone, Ethiopia, 2019.

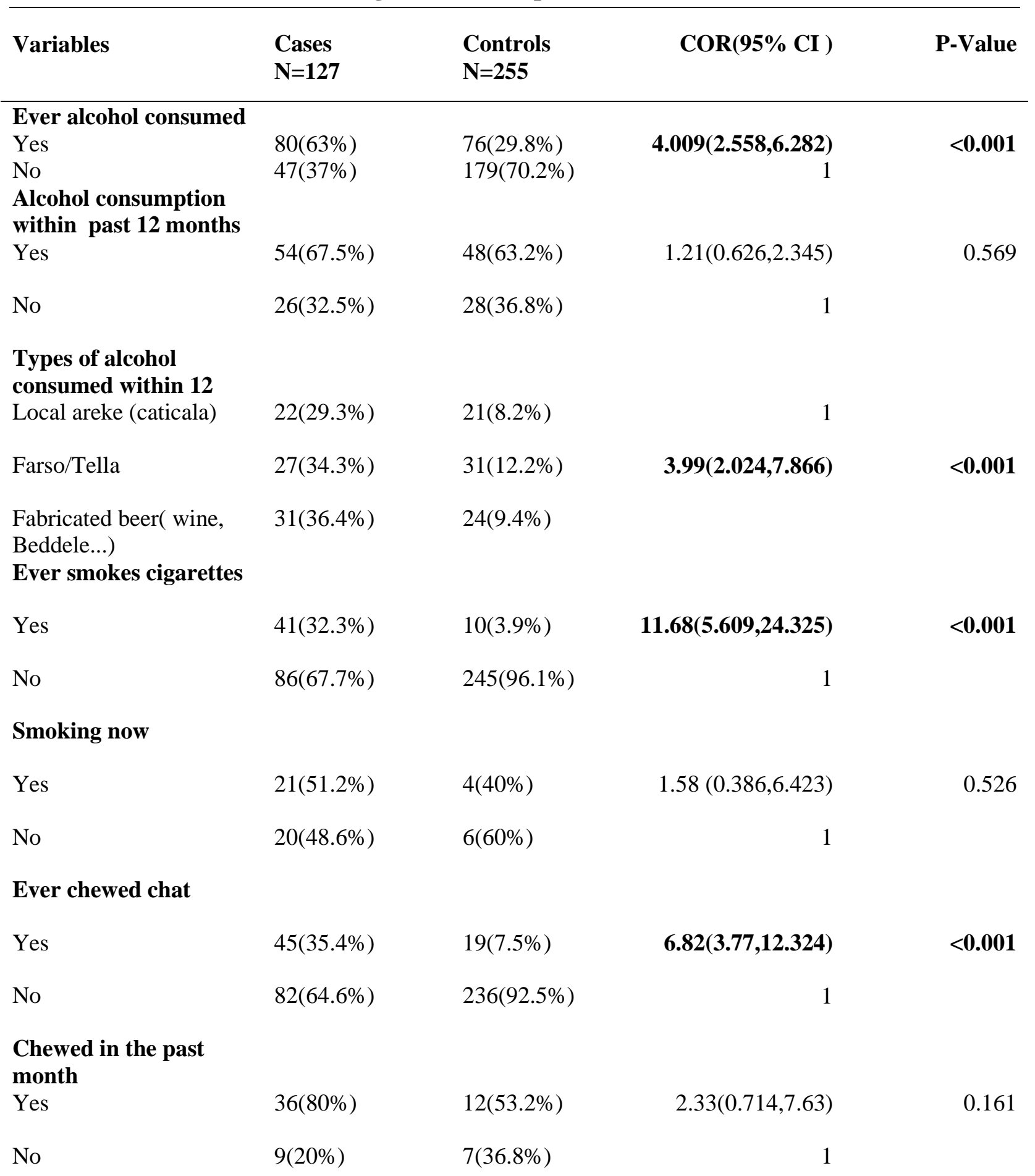


Table 2. Behavioral determinants of TB among HIV infected adults in public health facilities of Horro Guduru Wollega Zone, Ethiopia, 2019(continued).

\begin{tabular}{|c|c|c|c|c|}
\hline \multicolumn{5}{|l|}{$\begin{array}{l}\text { Ever Uses of } \\
\text { raw/uncooked milk }\end{array}$} \\
\hline No & $50(39.4 \%)$ & $193(75.7 \%)$ & 1 & \\
\hline \multicolumn{5}{|l|}{$\begin{array}{l}\text { Prisoned in the past } 2 \\
\text { years }\end{array}$} \\
\hline Yes & $43(33.9 \%)$ & $20(7.8 \%)$ & $6.02(3.347,10.808)$ & $<0.001$ \\
\hline No & $84(66.1 \%)$ & $235(92.2 \%)$ & 1 & \\
\hline \multicolumn{5}{|c|}{$\begin{array}{l}\text { Animals(cattle's and/or } \\
\text { pets) live with people } \\
\text { in the same House }\end{array}$} \\
\hline Yes & $60(47.2 \%)$ & $93(36.5 \%)$ & $1.56(1.013,2.402)$ & 0.044 \\
\hline No & $67(52.8 \%)$ & $162(63.5 \%)$ & 1 & \\
\hline \multicolumn{5}{|l|}{$\begin{array}{l}\text { Means of } \\
\text { transportation }\end{array}$} \\
\hline Foot & $29(22.8 \%)$ & $78(30.6 \%)$ & 1 & \\
\hline Car & $89(70.1 \%)$ & $149(58.4 \%)$ & $1.61(0.974,2.651)$ & 0.063 \\
\hline Horse back & $8(6.3 \%)$ & $22(8.6 \%)$ & $0.98(0.392,2.441)$ & 0.962 \\
\hline Bicycle & $1(0.8 \%)$ & $6(2.4 \%)$ & $0.43(0.052,3.885)$ & 0.466 \\
\hline \multicolumn{5}{|c|}{$\begin{array}{l}\text { Where usually go for } \\
\text { medical care when sick }\end{array}$} \\
\hline $\begin{array}{l}\text { Government clinic or } \\
\text { hospital }\end{array}$ & $102(80.3 \%)$ & 191(74.9\%) & $1.34(0.255,7.003)$ & 0.733 \\
\hline Private clinic & $19(15 \%)$ & $52(20.4 \%)$ & $0.91(0.163,5.111)$ & 0.918 \\
\hline Traditional healer & $4(3.1 \%)$ & $7(2.7 \%)$ & $1.43(0.184,11.085)$ & 0.733 \\
\hline Clinic run by a NGO & $2(1.6 \%)$ & $5(2 \%)$ & 1 & \\
\hline
\end{tabular}

COR: Crude Odds Ratio, CI: Confidence Interval, 1: reference category

Regarding current ART adherence, 76(59.8\%) of cases and 198(77.6\%) controls had good, $36(28.3 \%)$ of cases and 55(21.6\%) of controls had fair and 15(11.8\%) of cases and 2(0.8\%) of controls had poor adherence. All cases and controls were on ART during the study period and $25(19.7 \%)$ of cases and 23(9\%) of controls had history of loss of ART follow up. 
Table 3.ART adherence status among HIV infected adults in public health facilities of Horro Guduru Wollega Zone, Ethiopia, 2019.

\begin{tabular}{lllrr}
\hline Variables & $\begin{array}{l}\text { Cases } \\
\text { N=127 }\end{array}$ & $\begin{array}{l}\text { Controls } \\
\text { N=255 }\end{array}$ & COR(95\% CI ) & P-Value \\
\hline $\begin{array}{l}\text { Current ART } \\
\text { adherence }\end{array}$ & & & & \\
$\begin{array}{l}\text { Good }(>95 \%) \\
\text { Fair }(85-94 \%)\end{array}$ & $76(59.8 \%)$ & $198(77.6 \%)$ & 1 & \\
Poor $(<85 \%)$ & $15(11.8 \%)$ & $2(0.8 \%)$ & $\mathbf{1 9 . 5 4 ( 4 . 3 6 5 , 8 7 . 4 7 6 )}$ & $<$ \\
$\begin{array}{l}\text { Lost ART } \\
\text { follow up }\end{array}$ & & & & $<0.035$ \\
Yes & $25(19.7 \%)$ & $23(9 \%)$ & $\mathbf{0 . 0 0 1}$ \\
No & $102(80.3 \%)$ & $232(91 \%)$ & & \\
\hline
\end{tabular}

COR: Crude Odds Ratio, CI: Confidence Interval, 1: reference category

\section{Clinical determinants of TB among HIV infected Adults}

Among all respondents, 64(50.4\%) of cases and 51(20\%) of controls had $<11 \mathrm{~g} / \mathrm{dl}$ of $\mathrm{Hgb}$, while 63(49.6\%) of cases and $204(80 \%)$ of controls had $\mathrm{Hgb} \geq 11 \mathrm{~g} / \mathrm{dl}$. Of the total respondents, $56(44.1 \%)$ of cases and $40(15.7 \%)$ of controls had TB history, but $71(55.9 \%)$ of cases and $215(84.3 \%)$ of controls had not. From the cases $14(11 \%)$ and 24(18.9\%) were living with TB patient in the house and TB patient in neighbor's house respectively, but from the control group $3(1.2 \%)$ and $18(7.1 \%)$ were living with TB patient in the house and TB patient in neighbor's house respectively. 
Table 4. Clinical determinants of TB among HIV infected adults in public health facilities of Horro Guduru Wollega Zone, Ethiopia, 2019.

\begin{tabular}{|c|c|c|c|c|}
\hline Variables & $\begin{array}{l}\text { Cases } \\
\mathbf{N}=127\end{array}$ & $\begin{array}{l}\text { Controls } \\
\mathrm{N}=255\end{array}$ & COR $(95 \% \mathrm{CI})$ & P-Value \\
\hline \multicolumn{5}{|l|}{ Presence of OI } \\
\hline Yes & $86(67.7 \%)$ & $92(36.1 \%)$ & $3.72(2.367,5.836)$ & $<0.001$ \\
\hline No & $41(32.3 \%)$ & $163(63.9 \%)$ & 1 & \\
\hline \multicolumn{5}{|l|}{ WHO clinical stage } \\
\hline Stage I \& II & $38(29.9 \%)$ & $223(87.5 \%)$ & 1 & \\
\hline Stage III \& IV & $89(70.1 \%)$ & $32(12.5 \%)$ & $16.32(9.601,27.746)$ & $<0.001$ \\
\hline \multicolumn{5}{|l|}{$\begin{array}{l}\text { Initial CD4 cells } \\
\text { (cells/ } / \mathbf{L})\end{array}$} \\
\hline$<200$ & $54(42.5 \%)$ & $58(22.7 \%)$ & $2.39(1.35,4.245)$ & 0.003 \\
\hline $200-500$ & $45(35.5 \%)$ & $125(49 \%)$ & $0.93(0.532,1.611)$ & 0.785 \\
\hline$\geq 500$ & $28(22 \%)$ & $72(28.3 \%)$ & 1 & \\
\hline \multicolumn{5}{|l|}{ Recent CD4 cells } \\
\hline$<200$ & $28(22 \%)$ & $13(5.1 \%)$ & $6.32(3.015,13.238)$ & $<0.001$ \\
\hline $200-500$ & $54(42.5 \%)$ & $110(43.1 \%)$ & $1.44(0.9,2.303)$ & 0.128 \\
\hline$\geq 500$ & $45(35.4 \%)$ & $132(51.8 \%)$ & 1 & \\
\hline \multicolumn{5}{|l|}{ Recent viral load } \\
\hline$<1000$ (suppressed) & $89(70.1 \%)$ & $212(83.1 \%)$ & 1 & \\
\hline$\geq 1000$ (unsuppressed) & $38(29.9 \%)$ & $43(16.9 \%)$ & $2.11(1.275,3.477)$ & 0.004 \\
\hline \multicolumn{5}{|l|}{$\begin{array}{l}\text { Current ART } \\
\text { regimen provided }\end{array}$} \\
\hline $1^{\text {st }}$ line & $125(98.4 \%)$ & $250(98 \%)$ & 1 & \\
\hline $2^{\text {nd }}$ line & $2(1.6 \%)$ & $5(2 \%)$ & $0.8(0.153,4.182)$ & 0.791 \\
\hline \multicolumn{5}{|l|}{ Hemoglobin (g/dl) } \\
\hline$<11$ & $64(50.4 \%)$ & $51(20 \%)$ & $4.06(2.555,6.462)$ & $<0.001$ \\
\hline$\geq 11$ & $63(49.6 \%)$ & $204(80 \%)$ & 1 & \\
\hline \multicolumn{5}{|l|}{ BMI } \\
\hline$<18.5$ & $50(39.4 \%)$ & $42(16.5 \%)$ & $3.29(2.026,5.354)$ & $<0.001$ \\
\hline$\geq 18.5$ & $77(60.6 \%)$ & $213(83.5 \%)$ & 1 & \\
\hline \multicolumn{5}{|l|}{ History of TB } \\
\hline Yes & $56(44.1 \%)$ & $40(15.7 \%)$ & $4.24(2.607,6.895)$ & $<0.001$ \\
\hline No & $71(55.9 \%)$ & $215(84.3 \%)$ & 1 & \\
\hline \multicolumn{5}{|l|}{$\begin{array}{l}\text { Respiratory tract } \\
\text { infection in the past } \\
12 \text { months }\end{array}$} \\
\hline Yes & $92(72.4 \%)$ & $113(44.3 \%)$ & $3.30(2.083,5.238)$ & 0.26 \\
\hline No & $35(27.6 \%)$ & $142(55.7 \%)$ & 1 & \\
\hline
\end{tabular}


Table 4.Clinical determinants of TB among HIV infected adults in public health facilities of Horro Guduru Wollega Zone, Ethiopia, 2019( continued).

\section{Bronchial Asthma}

Yes

No

DM

Yes

No

HTN

Yes

No

TB patient in the house

Yes

No

TB patient in

Neighbors house

Yes

No
$29(22.8 \%) \quad 28(11 \%)$

$98(77.2 \%)$

$18(14.2 \%)$

109(85.8\%)

$9(3.5 \%)$

$246(96.5 \%)$

4.51(1.966,10.365)

1

$36(28.3 \%)$

91(71.7\%)

$26(10.2 \%)$

$229(89.8 \%)$

2.39(1.356,4.245)

1

0.003

COR: Crude Odds Ratio, CI: Confidence Interval, 1: reference category 


\section{Health facility related determinants of TB among HIV infected adults}

Eighty three $(65.4 \%)$ of cases and $171(67.1 \%)$ of controls took them less than an hour to reach health institution, $37(29.1 \%)$ of cases and 68(26.7\%) of controls took them Half a day and $7(5.5 \%)$ of cases and $16(6.3 \%)$ of controls took them One day and more. Ninety seven (76.4\%) of cases and 205(80.4\%) of controls were used IPT prophylaxis, but 30(23.6\%) of cases and $50(19.6 \%)$ of controls were not used. Of the total study participants, 113(89\%) of cases and 213 $(83.5 \%)$ of controls were used cotrimoxazole prophylaxis, while 14(11\%) of cases and $42(16.5 \%)$ of controls were not used. 
Table 5.Health facility related determinants of TB among HIV infected adults in public health facilities of Horro Guduru Wollega Zone, Ethiopia, 2019.

\begin{tabular}{|c|c|c|c|c|}
\hline Variables & $\begin{array}{l}\text { Cases } \\
\mathrm{N}=127\end{array}$ & $\begin{array}{l}\text { Controls } \\
\mathrm{N}=255\end{array}$ & $\operatorname{COR}(95 \% \mathrm{CI})$ & P-Valu \\
\hline \multicolumn{5}{|c|}{ Screened TB at initial ART visit } \\
\hline Yes & $109(85.8 \%)$ & $217(85.1 \%)$ & $1.06(0.58,1.94)$ & 0.85 \\
\hline No & $18(14.2 \%)$ & $38(14.9 \%)$ & 1 & \\
\hline \multicolumn{5}{|c|}{ Nearby health institution to take } \\
\hline Health center & $96(75.6 \%)$ & $197(77.3 \%)$ & 1 & \\
\hline Hospital & $31(24.4 \%)$ & $58(22.7 \%)$ & $1.09(0.665,1.808)$ & 0.717 \\
\hline \multicolumn{5}{|c|}{$\begin{array}{l}\text { Appointment for ART } \\
\text { medications }\end{array}$} \\
\hline Monthly & $72(56.7 \%)$ & $166(65.1 \%)$ & 1 & \\
\hline Every 3 month & $45(35.4 \%)$ & $78(30.6 \%)$ & $1.33(0.84,2.106)$ & 0.224 \\
\hline Every 6 month & $10(7.9 \%)$ & $11(4.3 \%)$ & $2.09(0.852,5.155)$ & 0.107 \\
\hline \multicolumn{5}{|c|}{ Time to reach health institution } \\
\hline Less than an hour & $83(65.4 \%)$ & $171(67.1 \%)$ & 1 & \\
\hline Half a day & $37(29.1 \%)$ & $68(26.7 \%)$ & $1.12(0.695,1.809)$ & 0.64 \\
\hline One day and more & $7(5.5 \%)$ & $16(6.3 \%)$ & $0.90(0.357,2.267)$ & 0.826 \\
\hline
\end{tabular}

Distance to reach health institution (Km)

$\begin{array}{lllll}<4 \mathrm{~km} & 35(27.6 \%) & 58(22.7 \%) & 0.44(0.212,0.902) & 0.025 \\ 4-9 \mathrm{~km} & 14(11 \%) & 53(20.8 \%) & 0.89(0.543,1.483) & 0.673 \\ >9 \mathrm{Km} & 78(61.4 \%) & 144(56.5 \%) & 1 & \\ \text { Cover mouth while coughing } & & & & \\ \text { Yes } & 65(51.2 \%) & 141(55.3 \%) & 1 & \\ \text { No } & 62(48.8 \%) & 114(44.7 \%) & 1.18(0.77,1.807) & \\ \text { Use of IPT prophylaxis } & & & & \\ \text { Yes } & 97(76.4 \%) & 205(80.4 \%) & 0.79(0.472,1.317) & 0.448 \\ \text { No } & 30(23.6 \%) & 50(19.6 \%) & 1 & \\ \text { Use of CPT } & & & & \\ \text { Yes } & 98(77.2 \%) & 216(84.7 \%) & 0.61(0.357,1.043) & 0.071 \\ \text { No } & 29(22.8 \%) & 39(15.3 \%) & 1 & \end{array}$

COR: Crude Odds Ratio, CI: Confidence Interval, 1: reference category 


\section{Determinants of TB/HIV co-infection}

In bivariable analysis a number of variable; age $\geq 35$, Sex, Income, No. of family members in the house, ever alcohol consumption, smoking, ever chew khat, ever use of raw milk, Prisoned in the past 2years, Fuel used for cooking, Living with animals in the same house, ART adherence, Loss of ART follow up, WHO clinical stage III\&IV, CD4 cells count <200, Viral load>1000, $\mathrm{Hgb}<11 \mathrm{~g} / \mathrm{dl}, \mathrm{BMI}<18.5 \mathrm{~kg} / \mathrm{m}^{2}$, History of TB, Asthma, DM, HTN, TB patient in the house, TB patient in the neighbor's house, Time of appointment for ART medications, Distance between home and health institution and use of CPT were significant at $\mathrm{p}$ value $<0.25$ and are potential candidates for the multiple logistic analysis.

In multiple logistic regression analysis, age $\geq 35$, ever alcohol consumption, ever chewing khat, imprisoned in the past 2 years, history of TB infection in the past, presence of TB patients in the house, WHO clinical stage III\& IV, Hgb level <11g/dl, CD4 cell count <200 were significantly associated with the development of TB/HIV co-infections while co-trimoxazole use were protective against TB/HIV co-infection.

Patients with age $\geq 35$ years were about two and half times more likely risky in developing TB/HIV co-infection than patients under the age of 35 years [AOR $=2.65 ; 95 \%$ CI (1.293, 5.444)]. Furthermore, Patients who had ever consumed alcohol had about three times higher risk

of developing TB/HIV co-infection than non-consumers [AOR=3.34; 95\% CI $(1.68,6.99)]$. HIV infected adults who ever chewed chat had five times higher risk of developing TB than those had not ever chewed $[\mathrm{AOR}=5.08 ; 95 \% \mathrm{CI}(1.95,13.23)]$.

The study also showed that CD4 cell count $<200$ [AOR=3.67; 95\% CI $(1.50,9.0)]$, Base line WHO clinical stage III \& IV [AOR=11.85; 95\% CI (5.742,24.462)], Low Hgb level(<11g/dl) $[\mathrm{AOR}=2.795 \% \mathrm{CI}(1.378,5.452)]$ were emerged as predictors of $\mathrm{TB} / \mathrm{HIV}$ co-infection. Cotrimoxazole use were protective against TB/HIV co-infection [AOR $=0.193 ; 95 \%$ CI $(0.81$, 0.461)]. 
Table 6. Determinants of TB/HIV co-infection among adults attending public health facilities of Horro Guduru Wollega Zone, Ethiopia, 2019.

\begin{tabular}{|c|c|c|c|c|}
\hline Variables & $\begin{array}{l}\text { Cases } \\
\mathbf{N}=127\end{array}$ & $\begin{array}{l}\text { Controls } \\
\mathrm{N}=255\end{array}$ & $\operatorname{COR}(95 \% \mathrm{CI})$ & $\operatorname{AOR}(95 \% \mathrm{CI})$ \\
\hline \multicolumn{5}{|l|}{ Age in years } \\
\hline $18-34$ & $46(36.2 \%)$ & $134(52.5 \%)$ & 1 & 1 \\
\hline$\geq 35$ & $81(63.8 \%)$ & $121(47.5 \%)$ & $1.950(1.259,3.020)$ & $2.65(1.293,5.444) *$ \\
\hline \multicolumn{5}{|l|}{ Sex } \\
\hline Male & $52(40.9 \%)$ & $84(32.9 \%)$ & $1.41(0.91,2.19)$ & $4.84(0.25,10.42)$ \\
\hline Female & $75(59.1 \%)$ & $171(67.1 \%)$ & 1 & 1 \\
\hline \multicolumn{5}{|l|}{ Ever smoke } \\
\hline Yes & $41(32.3 \%)$ & $10(3.9 \%)$ & $11.68(5.609,24.325)$ & $2.86(0.71,11.49)$ \\
\hline No & $86(67.7 \%)$ & $245(96.1 \%)$ & 1 & 1 \\
\hline
\end{tabular}

Ever alcohol consumed

Yes

No

Ever chewed chat

$\begin{array}{lllll}\text { Yes } & 45(35.4 \%) & 19(7.5 \%) & 6.816(3.77,12.324) & \mathbf{5 . 0 8}(\mathbf{1 . 9 5}, \mathbf{1 3 . 2 3}) * \\ \text { No } & 82(64.6 \%) & 236(92.5 \%) & 1 & 1\end{array}$

Imprisoned

in the past 2

years

$\begin{array}{lllll}\text { Yes } & 43(33.9 \%) & 20(7.8 \%) & 6.02(3.347,10.808) & \mathbf{4 . 2 2}(\mathbf{1 . 5 6 , 1 1 . 3 7}) * \\ \text { No } & 84(66.1 \%) & 235(92.2 \%) & 1 & 1 \\ \text { ory of TB } & & & & \\ & & & & \\ \text { Yes } & 56(44.1 \%) & 40(15.7 \%) & 4.24(2.607,6.895) & \mathbf{2 . 4 6 ( 1 . 2 0 5 , 5 . 0 3 0})^{*} \\ \text { No } & 71(55.9 \%) & 215(84.3 \%) & 1 & 1\end{array}$

TB patient in the house

$\begin{array}{lllll}\text { Yes } & 14(11 \%) & 3(1.2 \%) & 10.41(2.933,36.93) & \mathbf{1 4 . 9 ( 1 . 9 9 , 2 1 . 6 3 ) *} \\ \text { No } & 113(89 \%) & 252(98.8 \%) & 1 & 1\end{array}$

WHO clinical stage

$\begin{array}{lllll}\text { I \& II } & 38(29.9 \%) & 223(87.5 \%) & 1 & 1\end{array}$

III \& IV $\quad 89(70.1 \%) \quad 32(12.5 \%) \quad 16.322(9.601,27.746) \quad \mathbf{1 1 . 8 5}(\mathbf{5 . 7 4 , 2 4 . 4 6}) *$ 
Table 6. Determinants of TB/HIV co-infection among adults attending public health facilities of Horro Guduru Wollega Zone, Ethiopia, 2019 (continued).

\begin{tabular}{|c|c|c|c|c|}
\hline $\begin{array}{l}\text { ART } \\
\text { adherence } \\
\text { Good }(>95 \%)\end{array}$ & $76(59.8 \%)$ & $198(77.6 \%)$ & 1 & 1 \\
\hline Fair $(8594 \%)$ & $36(28.4 \%)$ & $55(21.6 \%)$ & $1.71(1.038,2.802)$ & $1.17(0.397,3.46)$ \\
\hline Poor $(<85 \%)$ & $15(11.8 \%)$ & $2(0.8 \%)$ & $19.54(4.365,87.476)$ & $9.22(0.74,11.51)$ \\
\hline \multicolumn{5}{|l|}{$\begin{array}{l}\text { CD4 at initial } \\
\text { diagnosis }\end{array}$} \\
\hline$<200$ & $54(42.5 \%)$ & $58(22.7 \%)$ & $2.39(1.35,4.245)$ & 3.67(1.5,0.9)* \\
\hline $200-500$ & $45(35.4 \%)$ & $125(49 \%)$ & $0.93(0.532,1.611)$ & $0.346(0.149,0.809)$ \\
\hline$\geq 500$ & $28(22 \%)$ & $142(55.7 \%)$ & 1 & 1 \\
\hline \multicolumn{5}{|l|}{ Temoglobin } \\
\hline$<11$ & $64(50.4 \%)$ & $51(20 \%)$ & $4.06(2.555,6.462)$ & $3.77(1.843,7.716) *$ \\
\hline$\geq 11$ & $63(49.6 \%)$ & $204(80 \%)$ & 1 & 1 \\
\hline \multicolumn{5}{|l|}{ HTN } \\
\hline Yes & $36(28.3 \%)$ & $26(10.2 \%)$ & $3.48(1.991,6.099)$ & $4.29(0.166,1.103)$ \\
\hline No & $91(71.7 \%)$ & $229(89.8 \%)$ & 1 & 1 \\
\hline \multicolumn{5}{|l|}{ BMI } \\
\hline$<18.5$ & $50(39.4 \%)$ & $42(16.5 \%)$ & $3.29(2.026,5.354)$ & $1.93(0.912,4.095)$ \\
\hline$\geq 18.5$ & $77(60.6 \%)$ & $213(83.5 \%)$ & 1 & 1 \\
\hline \multicolumn{5}{|l|}{$\begin{array}{l}\text { Distance from } \\
\text { health } \\
\text { institution }\end{array}$} \\
\hline$<4 \mathrm{~km}$ & $35(27.6 \%)$ & $58(22.7 \%)$ & $0.438(0.212,0.902)$ & $1.57(0.721,3.456)$ \\
\hline $4-9 \mathrm{~km}$ & $14(11 \%)$ & $53(20.8 \%)$ & $0.898(0.543,1.483)$ & $0.644(0.249,1.666)$ \\
\hline$>9 \mathrm{Km}$ & $78(61.4 \%)$ & $144(56.5 \%)$ & 1 & 1 \\
\hline \multicolumn{5}{|l|}{ Use of CPT } \\
\hline Yes & $98(77.2 \%)$ & $216(84.7 \%)$ & $0.61(0.357,1.043)$ & $0.193(0.81,0.461)^{*}$ \\
\hline No & $29(22.8 \%)$ & $39(15.3 \%)$ & 1 & 1 \\
\hline
\end{tabular}




\section{DISCUSSION}

The objective of this study was to identify determinants of TB infection among HIV infected adults.

The result of this study depicted that old aged PLWH was more likely to get TB coinfection. This finding is similar with study done in Brazil (23). This is possibly due to weakening of the immune system as age increases. However, Study conducted in western Ethiopia and German states that age was not associated with TB/HIV co-infection $(5,41)$. The difference is possibly explained by majority of the study participants in this study are those who are greater than the indicated age category and the difference of living standards between rich and poor countries which may have effect on nutritional status and exposure to disease; that is living in poor conditions such as food insecurity, inappropriately constructed house and lack of access to health care make them vulnerable to infection (42).

In this study, those who ever use alcohol were more likely to get TB co-infection in adults. This finding is similar with multicenter case control study done in North West Ethiopia, Nigeria, Amhara region (Ethiopia), India $(6,32,43,44)$. This could be explained by social mixing pattern which may increase the risk of exposure to active infectious TB disease in areas where alcohols mostly available like bars where people drinks in group (45), the other possible reason was alcohol consumption reduces the immune response in human and increases the risk for TB/HIV co-infection (2).Alcohol abuse is an important cause of immunological impairment (7).

The result of study shows that those who had ever chewed khat had five times more likely to develop TB/HIV co-infection than non-chewers. This is similar with studies conducted in North West Ethiopia (9). The possible reason was those who had chewed khat are most likely smoke cigarette and they may chew in group (creating overcrowdings) makes favorable condition for transmission of active tuberculosis.

Being imprisoned was significantly associated with TB/HIV co-infection development in this study. This finding is consistent with previous studies which say those who were imprisoned were at risk of TB/HIV co-infection (1). This can be explained by overcrowdings and enclosed space of the prisons that favors the transmission of TB. The other possible reason can be access to appropriate chemoprophylaxis in prisons can be problematic due to reasons such as affordability, circulation of suboptimal drugs and the low priority given to prison healthcare (46).

Other independent predictors of TB/HIV co-infection in this study were base line WHO clinical stage III \& IV. This result is consistent with other studies done in different parts of Ethiopia (5, $10,14,32$ ), Tanzania (47).This might be due to the fact that once patients get into late stages, the immune protective capacity will be minimal, making them predisposed to TB infection. This suggests that those who are in WHO stage III \& IV might be immune compromised and predisposed to TB (30). 
This study also showed that low CD4 cell count is risk for TB/HIV co-infection. HIV positive adults having CD4 cell count $<200$ cells $/ \mu \mathrm{L}$ were more likely to develop TB/HIV co-infection than those adults who had CD4 cells count $>500$ cell $/ \mu \mathrm{L}$. This finding is similar with previous studies $(2,6,9)$. This can be explained by decrement in CD4 lymphocyte leads the body's defense mechanism to be decreased exposing the individuals with HIV to develop various opportunistic infection like $\mathrm{TB}(13,43)$.

Having $\mathrm{Hgb}<11 \mathrm{~g} / \mathrm{dl}$ is significantly associated with the development of TB/HIV co-infection; those who had hemoglobin $<11 \mathrm{~g} / \mathrm{dl}$ were more than three and half times more likely to develop TB/HIV co-infection than those who had hemoglobin $\geqslant 11 \mathrm{~g} / \mathrm{dl}$ as this study revealed. This finding is concordant with studies conducted in North East Ethiopia, Addis Ababa, Jimma and Mettu $(6,30,39)$. TB and low hemoglobin level might be indirectly associated with advanced stage of HIV disease. When HIV positive patients have chronic disease and high viral load, it resulted in immune-suppression and suppression of red blood production in bone marrow. The other possible explanation might be malnutrition, side effects of medications, opportunistic infection and advanced stage of disease.

Individuals who had history of TB had greater risk of developing TB/HIV co-infection than those who had no previous history of TB as this study showed. This can be explained by poor compliance of anti-TB medications, reactivation of $\mathrm{TB}$ in individuals with the existing diminished immunity. This finding is in agreement with the study conducted in Northeast Ethiopia and Arbaminch (2, 6).

This study also revealed that presence of TB patients in the house had significantly associated with the development of TB/HIV co-infection. This finding is consistent with a study conducted in Gambia, Jimma \& Mettu $(31,39)$. This can be explained by long time exposure to person who is infected with TB can increase the transmission of the disease between the members of households.

This study also revealed that study participants who used cotrimoxazole (CPT) were less likely to develop TB/HIV co-infection than those participants who had not used CPT. This finding is similar with study conducted in Bahirdar (9), Addis Ababa (30). This can be explained by CPT has beneficial effects in enhancing CD4 count as well as reducing viral load (9).

This study revealed that low socio economic status was not significantly associated with TB/HIV co-infection. This finding is similar with Study conducted in Jima which showed that low monthly income was not associated with $\mathrm{TB}(8)$. However, it was significantly associated with TB/HIV co-infection in study conducted in Burkina Faso(20).The discrepancy can be explained by estimation of income based on monthly salary don't reflect actual income of individuals, in this study wealth index was done. 
It is a proven fact that tobacco smoking reduces the immune response in human and increases the risk for TB $(2,9)$.In this study smoking was assessed and the variable was risk factor (significant) at the univariate stage but was not predictor at the multivariate stage. This finding is similar with Study conducted in Burkina Faso(20), Jima and Mettu(8), Gambia(31) which revealed that smoking was not significantly associated with TB. This could be probably due to low prevalence of smokers in study population. There could also be due to a social desirability bias where by smokers denied their smoking status.

\section{Conclusion}

This study came up with being in old age, alcohol use, Chewing khat, Having CD4cells <200, Advanced WHO clinical stage, $\mathrm{Hgb}<11 \mathrm{~g} / \mathrm{dl}$, Imprisoned in the past 2 years, History of previous TB and living with TB patients in the same house were factors associated with TB/HIV coinfection. Using Cotrimoxazole had a protective effect from acquiring TB/HIV co-infection.

\section{Abbreviations and Acronyms}

AIDS, Acquired Immuno Deficiency Syndrome ; ART, Antiretroviral Therapy; CDC , Communicable Diseases Control; CPT, Cotrimoxazole Preventive Therapy; DM, Diabetes Mellitus; EPTB, Extra Pulmonary Tuberculosis; HAART, Highly Active Antiretroviral Therapy ; HIV, Human Immunodeficiency Virus; IPT, Isoniazid Preventive Therapy; PLHIV, People Living with HIV; RTI, Respiratory Tract Infection

\section{Ethical consideration}

Ethical approval was obtained from Wollega University Research Ethics and Review Committee (RERC) before its commencement. The aim of the study was explained and informed consent was obtained from the study participants. Permission letter was obtained from Shambu Hospital and Health Centers getting necessary information, record reviewing and interviewing concerned individuals resides in Health institutions.

\section{Acknowledgement}

We would like to thank Wollega University Institute of Health for support for the realization of this finding. We are also grateful for Health institutions for the provision of the needed data for our study. Special thanks and appreciation to all those who agreed to participate in this study, mainly respondents, data collectors and supervisors. 


\section{Consent for publication}

Not applicable

\section{Availability of data and materials}

Data will be available upon request from the corresponding author.

\section{Competing interests}

The authors declare that they have no competing interests

\section{Funding}

This research was funded by Wollega University.

\section{Authors' contributions}

All authors contributed in conception, data analysis, drafting and critically revising the paper and agreed to be accountable for all aspects of the work. All authors have reviewed and approved the submission of the manuscript.

\section{Authors' detail}

ZB: Zelalem Belay, MPH, Instructor, Nekemte Health Science College

BE: MPH, Lecturer, Department of Public Health, Wollega University

MT: MPH, Department of Public Health, Wollega University. 


\section{REFERENCES}

1. World Vision. Technical Guideline for Tuberculosis (TB) and TB-HIV Program Implementation WORLD VISION. 2017;1-28.

2. Mama M, Manilal A, Tesfa H, Mohammed H, Erbo E. Prevalence of Pulmonary Tuberculosis and Associated Factors Among HIV Positive Patients Attending Antiretroviral Therapy Clinic at Arba Minch General Hospital, Southern Ethiopia. Open Microbiol J. 2018;12(1):163-71.

3. World Health Organisation regional office for Africa; annual report communicable diseases cluster;2016;1-14

4. World Health Organization Regional Office for Africa; Cotonou TB declaration. 2018;(March):2018-21.

5. Melkamu H, Seyoum B, Dessie Y. Determinants of Tuberculosis infection among adult HIV positives attending clinical care in Western Ethiopia: A case-control study. AIDS Res Treat. 2013;2013:1-7.

6. Ahmed A, Mekonnen D, Shiferaw AM, Belayneh F, Yenit MK. Incidence and determinants of Tuberculosis infection among adult patients with HIV attending HIV care in North-East Ethiopia: A retrospective cohort study. BMJ Open. 2018;8(2):1-14.

7. Leonardo F. Jurado and Diana M. Palacios. Tuberculosis: A Risk Factor Approach. IntechOpen ,Open Access books Built by scientists, for scientists. Sch Enviromental Sci. 2012; Additional information is available at the end of the chapter. Available from http:// dx. doi. org /10.5772 / intech open.73538

8. Taha M, Deribew A, Tessema F, Assegid S, Duchateau L, Colebunders R. Risk Factors of Active Tuberculosis in People Living with HIV/AIDS in Southwest Ethiopia: A Case Control Study. Ethiopia. J Health Sci. 2011;21(2):131-9.

9. Alemu YM, Awoke W, Wilder-Smith A. Determinants for Tuberculosis in HIV-infected adults in Northwest Ethiopia:multicentre case-control Study. BMJ Open. 2016;6(4).

10. Fekadu S, Teshome W, Alemu G. Prevalence and determinants of Tuberculosis among HIV infected patients in South Ethiopia. J Infect Dev Ctries. 2015;9(8):898-904.

11. Rachow A, Ivanova O, Wallis R, Charalambous S, Jani I, Bhatt N, et al. TB sequel: 
Incidence, pathogenesis and risk factors of long-term medical and social sequelae of pulmonary TB.BMC Pulm Med. 2019 Jan 7;19(1).

12. World Health Organization. HIV-Associated Tuberculosis Fact Sheet. 2018;(September). Available from: http://www.who.int/tb/areas-of-work/tb-hiv/tbhiv_factsheet.pdf?ua=1

13. Negussie A, Debalke D, Belachew T, Tadesse F. Tuberculosis co-infection and its associated factors among People living with HIV/AIDS attending Antiretroviral therapy clinic in Southern Ethiopia: A facility based retrospective study. BMC Res Notes [Internet]. 2018;11(1):1-5. Available from: https://doi.org/10.1186/s13104-018-3530-3

14. UNAIDS. Joint United Nations Programme on HIV/AIDS factsheet. Tuberculosis and HIV — Progress towards the 2020 target. 2019; Available from:

http://www.unaids.org/sites/default/files/media_asset/tuberculosis-and-hiv-progresstowards-the-2020-target_en.pdf

15. UNAIDS. Joint United Nations Programme fact sheet on TUBERCULOSIS AND HIV/AIDS. 2017.

16. Andersen G,Gracia L, Jesus F, Eneique N-E, Pillar M-D, Santiago M. Changes in tuberculosis in human immunodeficiency virus infected patients in a Spanish tertiary hospital (1995-2013). Rev Esp Quimioter [Internet]. 2018;31(4):329-35. Available from:http://www.embase.com/search/results?subaction=viewrecord\&from=export\&id=L6 23487507

17. Saraceni V, Schwartz Benzaken A, Mendes Pereira GF, Andrade BK, Oliveira PB, Arakaki-Sanchez D, et al. Tuberculosis burden on AIDS in Brazil: A study using linked databases. PLoS One. 2018 Nov 1;13(11).

18. Dinic L, Akande P, Idigbe EO, Ani A, Onwujekwe D, Agbaji O, et al. Genetic determinants of drug-resistant tuberculosis among HIV-infected patients in Nigeria. J Clin Microbiol. 2012;50(9):2905-9.

19. Owiti P, Onyango D, Momanyi R, Harries AD. Screening and testing for tuberculosis among the HIV-infected: outcomes from a large HIV programme in western Kenya. BMC Public Health. 2019;19(1):1-11.

20. Méda ZC, Sombié I, Sanon OWC, Maré D, Morisky DE, Chen YMA. Risk factors of Tuberculosis infection among HIV/AIDS patients in Burkina Faso. AIDS Res Hum 
Retroviruses. 2013;29(7):1045-55.

21. Kaplan R, Hermans S, Caldwell J, Jennings K, Bekker LG, Wood R. HIV and TB coinfection in the ART era: CD4 count distributions and TB case fatality in Cape Town. BMC Infect Dis. 2018;18(1):1-9.

22. Martín-Echevarría E, Serrano-Villar S, Sainz T, Moreno A, Casado JL, Dronda F, et al. Development of tuberculosis in human immunodeficiency virus infected patients receiving antiretroviral therapy. Int J Tuberc Lung Dis. 2014;18(9):1080-4.

23. Saraceni V, Schwartz Benzaken A, Mendes Pereira GF, Andrade BK, Oliveira PB, Arakaki-Sanchez D, et al. Tuberculosis burden on AIDS in Brazil: A study using linked databases. PLoS One. 2018;13(11):1-14.

24. Pawlowski A, Jansson M, Sko“ ld M, Rottenberg ME, Ka“llenius G (2012) Tuberculosis and HIV Co-Infection. PLoS Pathog 8(2): e1002464. doi:10.1371/journal.ppat.1002464

25. Setegn E, Muchaye G, Aanimut A, DickVan S.. Tuberculosis treatment outcomes in Ethiopia from 2003 to 2016, and impact of HIV co-infection and prior drug exposure: A systematic review and meta-analysis. PLoS One. 2018;13(3):1-18.

26. Nuru A, Mamo G, Zewude A, Mulat Y, Yitayew G, Admasu A. Preliminary investigation of the transmission of Tuberculosis between farmers and their cattle in smallholder farms in northwestern Ethiopia : a cross sectional study. BMC Res Notes. 2017;1-7.

27. Amare D. Tuberculosis and HIV Co-infection among Patients on Tuberculosis Treatment at Fenote Selam District Hospital, Amhara Regional State, Northwest Ethiopia. Type Double Blind Peer Rev Int Res J Publ Glob Journals Inc. 2015;15(5). Available from: https://globaljournals.org/GJMR_Volume15/2-Tuberculosis-and-HIV-Co-infection.pdf

28. Ogbo FA, Ogeleka P, Okoro A, Olusanya BO, Olusanya J, Ifegwu IK, et al. Tuberculosis disease burden Nigeria. 2018;1-11.

29. Hiregoudar V, Raghavendra B, Karinagannavar A, Khan W, Kamble S, Goud T. Proportion and determinants of tuberculosis among human immunodeficiency viruspositive patients attending the antiretroviral therapy center attached to a Medical College in South India. J Fam Community Med. 2016;23(2):88.

30. Kibret KT, Yalew AW, Belaineh BG, Asres MM. Determinant Factors Associated with Occurrence of Tuberculosis among Adult People Living with HIV after Antiretroviral 
Treatment Initiation in Addis Ababa, Ethiopia: A Case Control Study. PLoS One. 2013;8(5):23-7.

31. Hill PC, Jackson-Sillah D, Donkor SA, Otu J, Adegbola RA, Lienhardt C. Risk factors for pulmonary tuberculosis: A clinic-based case control study in The Gambia. BMC Public Health. 2006;6:1-7.

32. Kuyinu YA, Mohammed AS, Adeyeye OO, Odugbemi BA, Goodman OO, Odusanya OO. Tuberculosis infection control measures in health care facilities offering TB services in Ikeja local government area, Lagos, South West, Nigeria. BMC Infect Dis. 2016;16(1):17. Available from: http://dx.doi.org/10.1186/s12879-016-1453-y

33. Escombe AR, Ticona E, Chávez-pérez V, Espinoza M, Moore DAJ. Improving natural ventilation in hospital waiting and consulting rooms to reduce nosocomial tuberculosis transmission risk in a low resource setting. 2019;1-7.

34. Kigozi NG, Heunis JC, Engelbrecht MC, Rensburg APJ Van, Rensburg HCJD Van. Tuberculosis knowledge, attitudes and practices of patients at primary health care facilities in a South African metropolitan : research towards improved health education. $2017 ; 1-8$.

35. ENHARI/CDC TB/HIV surveillance team. National TB / HIV Sentinel Surveillance One year Report. 2013; ( (July 2011 - June 2012).

36. Senthilkumar M, Nadu T. International Journal of Pharmaceutical Sciences and Research. Ijspr. 2015;6(6):2496-503.

37. Abraham B, Selamu K, Tesfa T, Getachew A. Poor treatment outcomes and its determinants among tuberculosis patients in selected health facilities in East Wollega, Western Ethiopia. PLoS One. 2018;13(10):1-15. Available from: http://www.embase.com/search/results?subaction=viewrecord\&from=export\&id=L62455 5297\%0Ahttp://dx.doi.org/10.1371/journal.pone.0206227

38. Alemu YM, Awoke W, Wilder-Smith A. Determinants for tuberculosis in HIV-infected adults in Northwest Ethiopia:multicentre case-control Study. BMJ Open. 2016;6(4):1-6.

39. Taha M, Deribew A, Tessema F, Assegid S, Duchateau L, et al. Risk factors of active Tuberculosis in people living with HIV/AIDS in Southwest Ethiopia: a case control study

40. Global Adult Tobacco Survey Collaborative. Tobacco Questions for survey :a subset of 
key questions from the Global Adults T obacco Survey (GATS), $2^{\text {nd }}$ edition. Atlant GA; Centers for Disease Control and Prevention,2011.

41. Karo B, Haas W, Kollan C, Gunsenheimer-Bartmeyer B, Hamouda O, Fiebig L, et al. Tuberculosis among people living with HIV/AIDS in the German ClinSurv HIV Cohort: Long-term incidence and risk factors. BMC Infect Dis. 2014;14(1):1-12. Available from: BMC Infectious Diseases

42. Okhov B. Inequality in the global incidence and prevalence of tubercul losis ( TB ) and TB / HIV acording to the human development ind. 2019;

43. Mitku AA, Dessie ZG, Muluneh EK, Workie DL. Prevalence and associated factors of TB/HIV co-infection among HIV infected patients in Amhara region, Ethiopia. Afr Health Sci. 2016;16(2):588-95.

44. Hiregoudar V, Raghavendra B, Karinagannavar A, Khan W, Kamble S, Goud T. Proportion and determinants of Tuberculosis among human immunodeficiency viruspositive patients attending the antiretroviral therapy center attached to a Medical College in South India. J Fam Community Med. 2016;23(2):88.

45. Lönnroth K, Williams BG, Stadlin S, Jaramillo E, Dye C. Alcohol use as a risk factor for tuberculosis - A systematic review. BMC Public Health. 2008;8.

46. Edge CL, King EJ, Dolan K, Mckee M. Review article Prisoners co-infected with Tuberculosis and HIV : a systematic review. 2016;1-19.

47. Said K, Verver S, Kalingonji A, Lwilla F, Mkopi A, Charalambous S, et al. Tuberculosis among HIVHIV-infected population: Incidence and risk factors in rural Tanzania. Afr Health Sci. 2017;17(1):208-15. 